\title{
Heat transfer model of an iCVD reactor
}

\author{
R. Bakker*, V. Verlaan, A.D. Verkerk, C.H.M. van der Werf, L. van Dijk, H. Rudolph, J.K. Rath, R.E.I. Schropp \\ Utrecht University, Faculty of Science, Debye Institute for Nanomaterials Science, Nanophotonics - Physics of Devices, P.0. Box 80.000, 3508 TA Utrecht, The Netherlands
}

\section{A R T I C L E I N F O}

Available online $\mathrm{xxxx}$

\section{Keywords:}

Hot-wire CVD

iCVD

Heat transfer

\begin{abstract}
A B S T R A C T
Contrary to conventional HWCVD, the power consumption in the iCVD process is dominated by heat conduction rather than radiation. This is due to the fact that while the typical wire temperature for HWCVD is about $1750-2200{ }^{\circ} \mathrm{C}$, for iCVD the temperature is only $250-500{ }^{\circ} \mathrm{C}$. Typical deposition pressures are in the transition regime between the collision free regime, where the conduction is pressure dependent, and the collision mediated regime, where the conduction is pressure independent. The power loss due to heat conductivities of molecular nitrogen, glycidyl methacrylate (GMA) and tert-butylperoxide (TBPO) gases have been determined experimentally for these pressure regimes. The necessary power input to the filaments can be explained to be due to mainly heat dissipation by radiation and by gas conduction. This means that the dissociation process requires only very little power, about $2 \%$ of the total power consumption in a typical iCVD process.
\end{abstract}

(c) 2009 Elsevier B.V. All rights reserved.

\section{Introduction}

Initiated chemical vapor deposition (iCVD) is a gas-phase technique for depositing polymer films without the use of a solution or plasma and it can quite easily be integrated in large-scale device production [1]. In the iCVD process, metal wires are resistively heated to a temperature of typically $250{ }^{\circ} \mathrm{C}$, so that the initiator is decomposed into radicals. These radical species then initiate a free radical polymerization of monomers to form a film on a cooled substrate $\left(<25{ }^{\circ} \mathrm{C}\right)$. One of the polymers that can be deposited using iCVD is poly-glycidyl methacrylate (PGMA) [2,3]. Because no plasma is involved and the process temperatures are very low, the occurrence of any unwanted side reaction is minimized, resulting in a high purity of the deposited polymer $[3,4]$. Furthermore, the iCVD process consumes very little energy, due to the relatively low process temperatures, which is an advantage for implementation in commercial production lines.

Although for typical HWCVD processes that use filament temperatures in the $1750-2200{ }^{\circ} \mathrm{C}$ range, several studies have been done to examine the consumed power [5-7], no studies have, to our knowledge, been done for iCVD. It is obvious that at high temperatures and low pressures of a typical HWCVD process, heat conduction by the process gases has only a minor influence on the consumed power, compared to radiation. This difference is mainly due to the fact that radiation power is proportional to the fourth power of the wire temperature $\left(\mathrm{T}_{\mathrm{w}}^{4}\right)$, whereas the temperature dependence of conduction is of a lower order. In this paper, the power consumption of an iCVD reactor is studied.

\footnotetext{
* Corresponding author.

E-mail address: R.Bakker@uu.nl (R. Bakker).
}

\section{Experimental}

The investigated iCVD system contains a nichrome wire $(80 \% \mathrm{Ni}$, $20 \% \mathrm{Cr}$ ) mounted in a parallel array, $28 \mathrm{~mm}$ above the substrate in a home-built reactor (the PANDA), after a design by Prof. Gleason's laboratory at MIT. The reactor is cylindrically shaped and has an internal diameter of $25 \mathrm{~cm}$ and internal height of $5 \mathrm{~cm}$. The monomer, GMA ( $97 \%$, Aldrich) was heated in a glass chamber to a temperature of $60{ }^{\circ} \mathrm{C}$, and fed into the reactor through a pipe heated to $90{ }^{\circ} \mathrm{C}$. The initiator, tert-butyl peroxide (TBPO) of 98\% purity (Aldrich) was vaporized at room temperature. The initiator/precursor flow was controlled by metering valves. The substrate temperature was kept below $25{ }^{\circ} \mathrm{C}$ by means of water cooling on the back of the substrate holder. Current, voltage and temperature measurements were done using 175 True RMS multimeters and an 80TK Thermocouple Module (Fluke). Pressure was controlled by an MKS 615C pressure controller.

\section{Results and discussion}

In thermal equilibrium, the power supplied by the power supply is the total input power dissipated in resistively heated wires which can be described by the following expression:

$P_{\text {input }}=P_{\text {rad }}+P_{\text {cond }}+P_{\text {conv }}+P_{\text {re }}=I \cdot V$

where $P_{\text {rad }}$ is the radiative power loss, $P_{\text {cond }}$ is the power lost by conduction of the gas, $P_{\text {conv }}$ is the power loss through convective heat transfer and $P_{\text {re }}$ is the power that is consumed by the chemical reactions at the wires. Experiments in which we varied the gas flow rate showed no alterations in the power consumption. Furthermore, the heat conductivity at higher pressures saturates towards a constant 


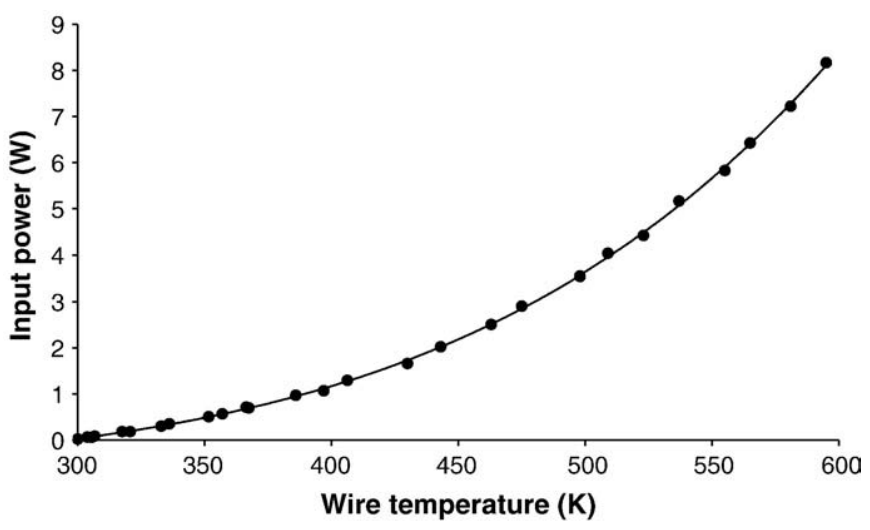

Fig. 1. The input power of the filament in vacuum versus the wire temperature. The line is a fit with the Stefan-Boltzmann radiation law (Eq. (1)).

value (see below). This means that convective heat transfer is only small and can be neglected.

In Fig. 1, the power input to the filaments in thermal equilibrium is given at the vacuum base pressure $(0.01 \mathrm{mbar})$ for various wire temperatures. At this low pressure, the effects of heat conduction are minimized. The power loss through blackbody radiation is described by the Stefan-Boltzmann radiation law:

$P_{\text {rad }}=P_{v a c}=\varepsilon \sigma_{S B} A_{w}\left(T_{w}^{4}-T_{s}^{4}\right)$

in which $\sigma_{\mathrm{SB}}$ is the Stefan-Boltzmann constant, $\varepsilon$ the emissivity of the system (the emissivity of the wire being the relevant value), $A_{w}$ is the surface area of the wire, $T_{w}$ its temperature and $T_{s}$ the temperature of the surroundings (the walls of the reactor). The measurements (dots) are fitted with Eq. (2) and the proper emissivity $\varepsilon$ was determined, and amounted to 0.31 , which corresponds reasonably with values found in literature for nichrome alloys with $80 \% \mathrm{Ni}(0.36)$ [8], the material used in our experiments.

The heat conduction from the hot wire to the wall of the reactor through the gas is dependent on the type of gas, the pressure, the temperature of the wire and geometry of the reactor. In our case, the reactor walls are cooled with water to maintain a constant temperature $\left(T_{\mathrm{s}}\right)$. Keeping the temperature of the wire $\left(T_{\mathrm{w}}\right)$ constant $\left(100 \pm 5^{\circ} \mathrm{C}\right)$ and well below the temperature at which TBPO starts to dissociate significantly $\left(150{ }^{\circ} \mathrm{C}\right)$ [9] allows us to study the pressure dependence of the heat conduction of GMA and TBPO.

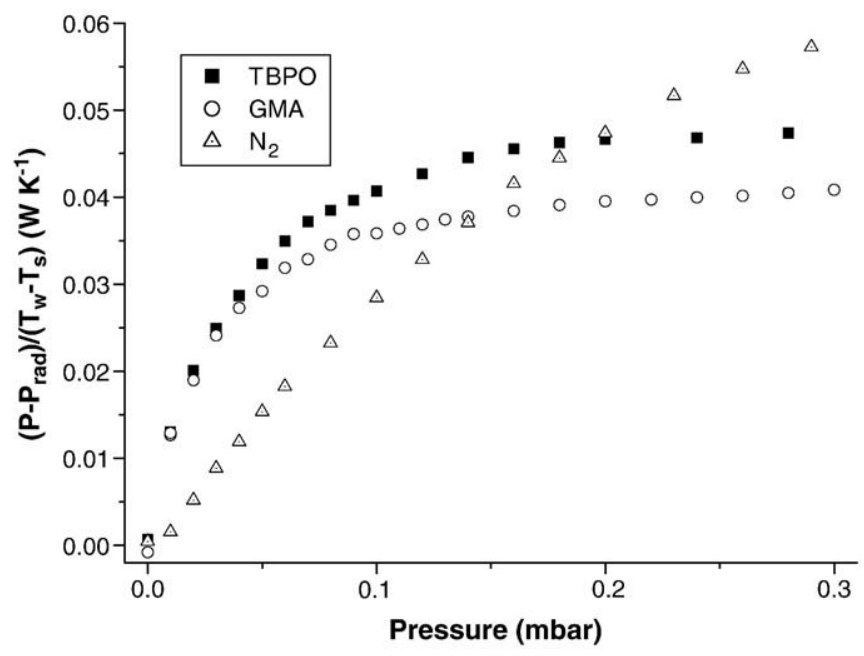

Fig. 2. The pressure dependence of the heat conduction for the relevant gases. (a)

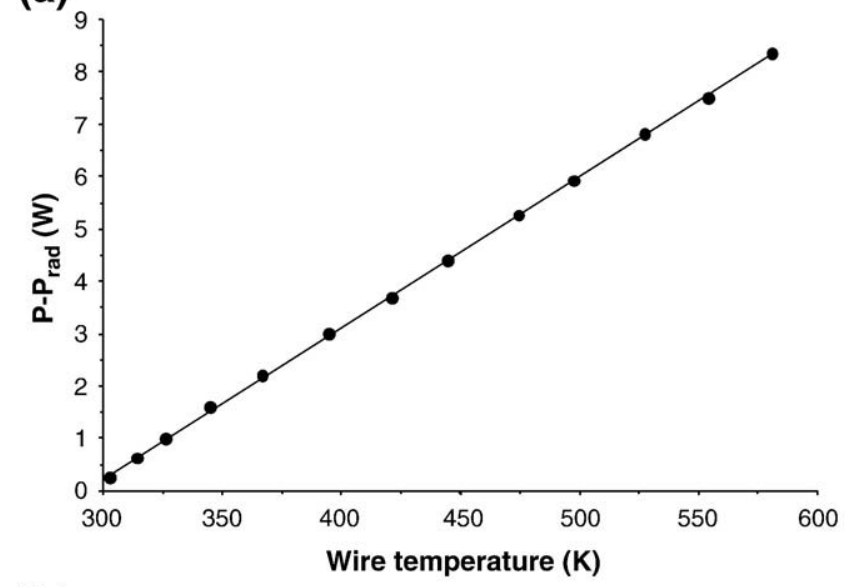

(b)

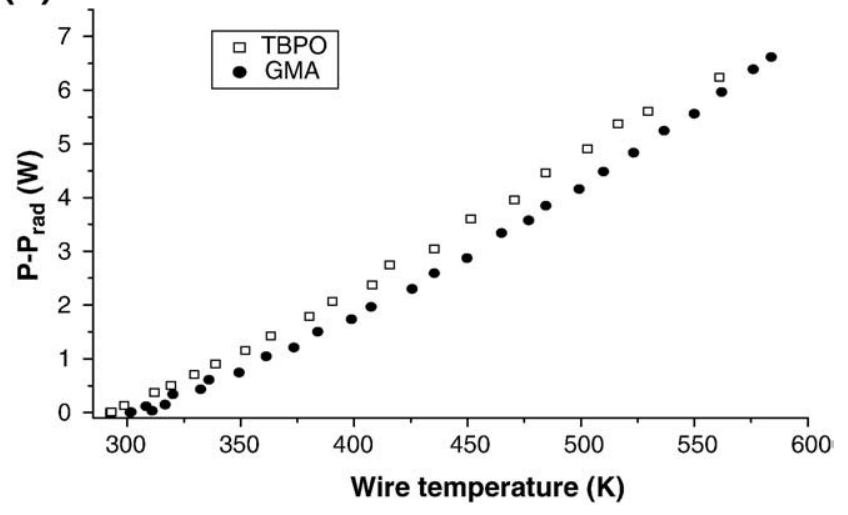

Fig. 3. The power consumed by heat conduction of (a) $\mathrm{N}_{2}$ at 0.10 mbar (the line is a linear fit) and (b) TBPO and GMA at 0.02 mbar.

Heat conduction takes place via different mechanisms in different pressure regimes. An important parameter in studying the pressure dependence of the heat conduction is the mean free path:

$\lambda=k T / p \sigma_{c} \sqrt{ } 2$,

in which kis the Boltzmann constant, p the pressure and $\sigma_{\mathrm{c}}$ the collision cross-sectionof themolecule.Athighpressures, forwhichthemeanfree pathofamoleculeissmallerthanthetypicalsizeofthereactor $\mathrm{D}(\lambda<<\mathrm{D})$, heat conduction is independent of pressure [10]. At low pressures $(\lambda>>D)$, the heat conduction is linearly dependent on the pressure $[10,11]$. Dividing the measured conductive power $\left(P-P_{\mathrm{vac}}\right)$ by $T_{\mathrm{w}}-T_{\mathrm{s}}$ corrects for the small fluctuations in the wire temperature. Fig. 2 shows the pressuredependence of conductive powerlossof $\mathrm{N}_{2}$,TBPOandGMA gases at the intermediate pressure range. Nitrogen gas is the only one of these gases for which the heat conduction does not saturate in the measured pressure regime ( $0-0.3 \mathrm{mbar})$.

Fig. 3a shows the power loss by conduction of 0.10 mbar $_{2}$ versus the wire temperature. As can be seen from Fig. 2, this is well within the pressure dependent regime (see Fig. 2). Conductive power is linearly dependent on $T_{\mathrm{w}}-T \mathrm{~s}$, as predicted by the theory for low-pressure conduction [10]. For GMA and TBPO, the temperature dependence at lower pressures (shown in Fig. $3 \mathrm{~b}$ ), studied at a relatively lower pressure regime $(0.02 \mathrm{mbar})$ where the conduction is pressure dependent), seems to deviate slightly from this linear behavior. Since the deviation is comparable for both gases and GMA does not dissociate at these relatively low temperatures, it is unlikely that it is due to any chemical reaction. 


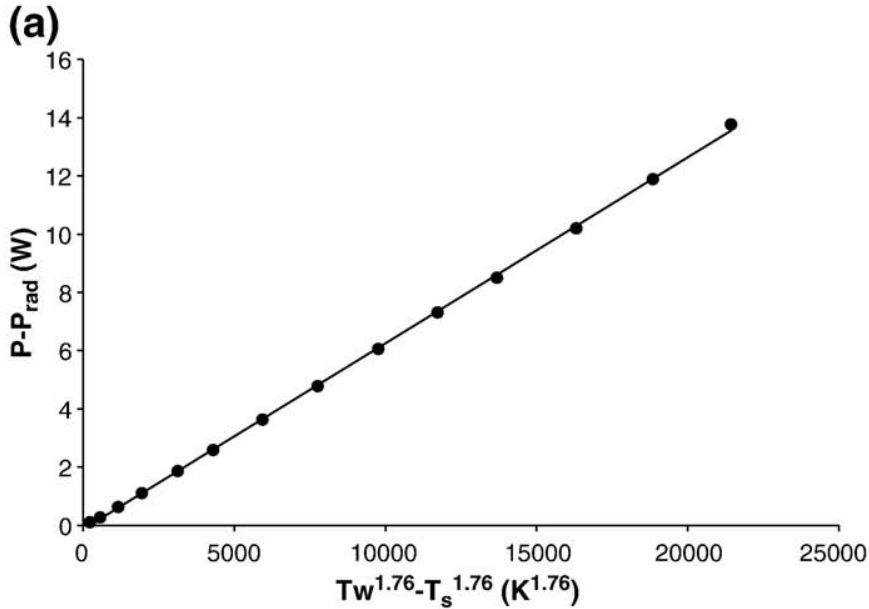

(b)

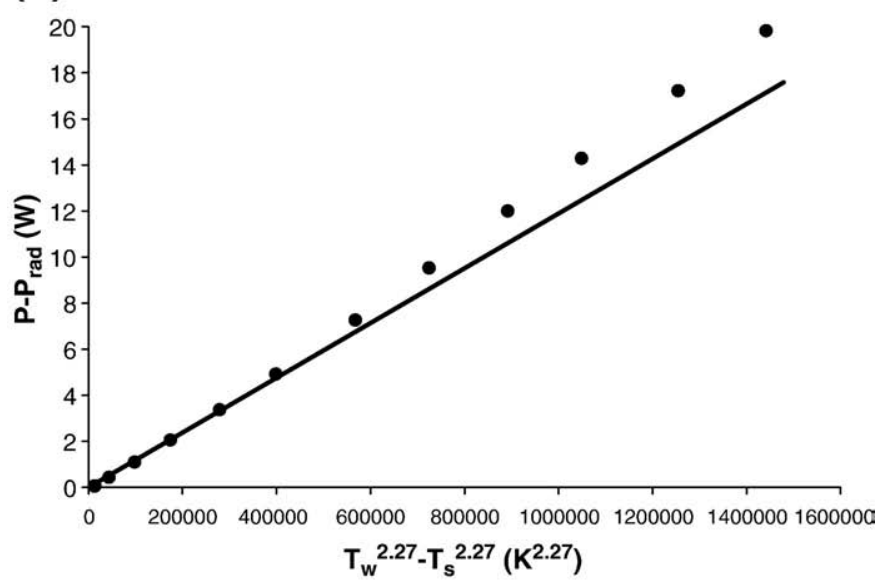

Fig. 4. The power consumed by heat conduction versus $\left(T_{w}^{(1+b)}-T_{s}^{(1+b)}\right)$ for (a) 4.0 mbar $\mathrm{N}_{2}$ pressure (including a linear fit) and (b) 0.20 mbar TBPO pressure (including a linear fit with the first seven data points).

The part of the heat capacity $\left(C_{\mathrm{v}}\right)$ of TBPO that is due to translation, rotation and vibration (and not dissociation of the peroxide bond), can be calculated from data for 2,2,5,5 tetramethylhexane, introducing compensating factors for replacing a $\mathrm{CH}_{2}$-goup by an oxygen atom [12] and can be expressed as a power function:

$$
C_{v, \text { calc }}(T)=a \cdot T^{b}=2.68 T^{0.77} \quad\left(J K^{-1} \mathrm{~mol}^{-1}\right)
$$

As the conduction power loss of both gases seems proportional to $\sim \mathrm{T}^{1.8}$ (as fitted from data in Fig. 3b) this indicates an influence of the temperature dependence of the heat capacity, which is $\sim \mathrm{T}^{0.77}$ for TBPO. This would mean that the power that is actually used to dissociate the molecule into radicals, which is the driving force of iCVD deposition [3], is negligible. These measurements contradict the statement in vacuum textbooks that the conductive power is linearly related to $\mathrm{T}_{\mathrm{w}}-\mathrm{T}_{\mathrm{s}}$ at low pressures [11].

At larger pressures $(\lambda<<D)$, heat conduction is dependent on the temperature of the wire and the walls, since the gas is dense enough to attain the thermal energy of the wire and thus its temperature. Using data from literature [13] for the thermal conductivity coefficient of $\mathrm{N}_{2}$ $\left(\kappa_{\mathrm{N} 2}\right)$ at temperatures between 290 and $620 \mathrm{~K}$ and a pressure of $1 \mathrm{~atm}$., the relation between $\mathrm{T}$ and $\kappa_{\mathrm{N} 2}$ was fitted with a power function to obtain:

$\kappa_{N_{2}}(T)=a \cdot T^{b}=0.35 \cdot 10^{-3} T^{0.76}(\mathrm{~W} / \mathrm{Km})$
Substituting Eq. (5) in the Fourier law of heat conduction [14] results in the following solution:

$P_{\text {cond }}=\frac{g}{(1+b)} \cdot a \cdot\left(T_{w}^{1+b}-T_{s}^{1+b}\right)=\frac{g}{(1+b)}\left(\kappa\left(T_{w}\right) \cdot T_{w}-\kappa\left(T_{s}\right) \cdot T_{s}\right)$

in which $g$ is an overall geometry term, which is typical for the reactor and $b$ is the exponent from Eq. (5). From this expression, the relation between the conductive power and the wire temperature is not linear but almost quadratic, as can be seen in Fig. 4a. In this graph, the conductive power is plotted against the $(1+b)^{\text {th }}$ power of the wire temperature. At high pressures, the plot yields a straight line.

When measuring at higher pressure for our process gas TBPO, we can use this expression for its conductivity coefficient:

$\kappa_{\text {dense }}=\frac{1}{3} \cdot \lambda \cdot C_{v} \cdot \bar{v} \cdot n$

in which $C_{\mathrm{v}}$ is the heat capacity, $\bar{v}$ the average velocity and $\mathrm{n}$ the molar density of the gas. The factor $1 / 3$ in this expression is in practice often compensated by a "reality factor" [11] or replaced by $25 \pi / 64$ [10], but since it is a constant value it does not change our model significantly. For an ideal gas, Eq. (7) becomes:

$\kappa_{\text {dense }}=\frac{2 \sqrt{R T} \cdot C_{v}}{3 \sigma_{c} N_{A} \sqrt{\pi M}}$

in which $R$ is the gas constant and $M$ the molar mass of the molecule. Using Eq. (4) for the heat capacity of TBPO, we can calculate the temperature dependence of the conductive power. Because there is also an extra factor $\mathrm{T}^{0.5}$ in the expression for $\kappa$, it is proportional to $\mathrm{T}^{1.27}$. This means that the conductive power becomes proportional to $\mathrm{T}^{2.27}$. Plotting the conductive power loss of $0.2 \mathrm{mbar}$ TBPO against $\left(\mathrm{T}_{\mathrm{w}}^{2.27}-\mathrm{T}_{\mathrm{s}}^{2.27}\right)$ gives a straight line, although there is some deviation (Fig. 4b). This deviation could be ascribed to power consumed by the dissociation reaction. The dissociation power can in this case be estimated to be as low as $2 \mathrm{~W}$ at $250{ }^{\circ} \mathrm{C}$. For deposition conditions, the partial TBPO pressure is about 4 times as low [2]. This means that under deposition conditions, the dissociation power is about $0.5 \mathrm{~W}$. If an estimate is made of the radiative power to be about $6 \mathrm{~W}$ at $523 \mathrm{~K}$ (Fig. 1) and the conductive power to be about $14 \mathrm{~W}$ (from conduction measurements at deposition conditions), the reaction power accounts for about $2 \%$ of the total power consumed in the process.

\section{Conclusions}

The power consumption for an iCVD reactor can be explained almost entirely by radiative and conductive heat transfer only. In the high pressure regime $(\lambda<<D)$, existing heat conduction theories can explain the results. At low pressures $(\lambda>>D)$, the heat capacity of the gases entails a certain temperature dependence of the heat conductivity not accounted for in common heat transfer theories. The higher order temperature dependence $\left(\mathrm{T}^{2.27}\right)$ of the conductive power of polyatomic gases indicates that heat conduction might not be as insignificant in other HWCVD processes as it is generally assumed.

\section{Acknowledgements}

We thank Prof. Karen Gleason's group at MIT for fruitful interaction.

\section{References}

[1] M. Gupta, K.K. Gleason, Thin Solid Films 515 (4) (2006) 1579.

[2] R. Bakker, V. Verlaan, C.H.M. Van der Werf, J.K. Rath, K.K. Gleason, R.E.I. Schropp, Surf. Coat. Technol. 201 (2007) 9422.

[3] Y. Mao, K.K. Gleason, Langmuir 20 (2004) 2484.

[4] K. Chan, K.K. Gleason, Chem. Vap. Depos. 11 (2005) 437.

[5] C.H.M. Van der Werf, P.A.T.T. Van Veenendaal, M.K. Van Veen, A.J. Hardeman, M.Y.S. Rusche, J.K. Rath, R.E.I. Schropp, Thin Solid Films 515 (3) (2006) 1117. 
[6] E. Zeiler, S. Schwartz, S.M. Rosiwal, R.F. Singer, Mat. Sci. Eng. A 335 (2002) 236

[7] T. Hata, H. Nakayama, Thin Solid Films 516 (2008) 558.

[8] K. Schaefers, M. Rösner-Kuhn, M.G. Frohberg, Int. J. Thermophys. 16-4 (1995) 997.

[9] K.S. Lau, Y. Mao, H.G. Pryce Lewis, S.K. Murthy, B.D. Olsen, L.S. Loo, K.K. Gleason, Thin Solid Films 501 (2006) 211.

[10] W. Kauzman, Kinetic theory of gases, W.A. Benjamin Inc., 1966, p. 206.
[11] E.P.Th.M. Suurmeijer, Th. Mulder, J. Verhoeven, Basisboek vacuumtechniek, NEVAC, 2000, p. 55.

[12] D.R. Stull, E.F. Westrum, G.C. Sinke, The chemical properties of organic compounds, John Wiley \& Sons, New York, 1969.

[13] Handbook of chemistry and physics, The Chemical Rubber Co., Cleveland, 1970

[14] A. Bejan, Heat transfer, John Wiley \& sons, New York, 1993. 